\title{
Caractérisation des Vergers de Manguiers (Mangifera indica L.) en Basse Casamance, Sénégal
}

\author{
Ousmane Ndiaye, \\ Urbain Diatta, \\ Madiba Nibaly,
}

Université Assane Seck de Ziguinchor,

Département d'Agroforesterie, Sénégal

Saliou Djiba,

Institut Sénégalais de Recherches Agricoles,

ISRA/Dijbelor, Ziguinchor, Sénégal

Kémo Badji,

Projet de Lutte contre les Mouches des Fruits PLMF/CEDEAO

Saliou Ndiaye,

Ecole Nationale Supérieure d'Agriculture, Université de Thiès, Sénégal

Doi:10.19044/esj.2020.v16n12p338 URL:http://dx.doi.org/10.19044/esj.2020.v16n12p338

\section{Résumé}

Les vergers à base de manguiers (Mangifera indica L.) en Casamance abritent des systèmes de culture particuliers. Ces systèmes de culture souffrent des attaques d'un cortège de ravageurs et maladies dont l'incidence serait associée à la diversité variétale du manguier, des espèces fruitières associées mais aussi à l'organisation fonctionnelle des vergers. Le présent travail a porté sur la détermination des caractéristiques et la typologie des plantations de manguiers. L'étude a été réalisée entre 2016 et 2018 dans trois Arrondissements du Département de Bignona (Kataba 1, Tendouck et Tenghory). La caractérisation structurale et l'étude des systèmes de production à base de manguiers ont été faites suite à des inventaires et des enquêtes et à partir de méthodes d'analyses multi variées et de classification sur des données provenant d'enquête auprès des planteurs et de mesures directes sur le terrain dans 217 vergers échantillonnés soit 44,28\%. La presque totalité des vergers de la zone est de type traditionnel avec des superficies comprises entre 0,2 et 10,7 ha. Le labour et le désherbage constituent les entretiens mécaniques les plus fréquents. Au total, 36 variétés de manguiers ont été notées (dont 31 variétés locales). Tous les trois Arrondissements restent dominés par des variétés comme la Keitt et la Kent. A cet effet, l'inventaire de la flore fruitière a permis d'identifier 46 espèces réparties dans 42 genres appartenant à 29 
familles dans l'ensemble des vergers. Ainsi, la typologie a permis de définir quatre groupes de vergers.

Mots-clés: Caractérisation, Verger, Mangifera indica, Casamance

\title{
Characterization of Mango Orchards (Mangifera indica L.) in Basse Casamance, Senegal
}

\author{
Ousmane Ndiaye, \\ Urbain Diatta, \\ Madiba Nibaly,
}

Université Assane Seck de Ziguinchor,

Département d'Agroforesterie, Sénégal

Saliou Djiba,

Institut Sénégalais de Recherches Agricoles,

ISRA/Dijbelor, Ziguinchor, Sénégal

Kémo Badji,

Projet de Lutte contre les Mouches des Fruits PLMF/CEDEAO

Saliou Ndiaye,

Ecole Nationale Supérieure d'Agriculture, Université de Thiès, Sénégal

\begin{abstract}
The orchards made from mango trees (Mangifera indica L.) in Casamance are home to particular cropping systems. These cropping systems suffer from attacks due to pests and diseases outbreak. This incidence might not only be associated with a diversity of mango trees and fruit species, but also with the functional organization of orchards. This paper focuses on determining the characteristics and typology of mango plantations. The study was carried out between 2016 and 2018 in three districts of the Department of Bignona (Kataba 1, Tendouck and Tenghory). The structural characterization and the study of mango-based production systems were carried out following inventories and surveys. It was also done using multi-varied analysis and classification methods based on data obtained from planters and direct measurements on the land in 217 orchards sampled (44.28\%). Almost all the orchards in the area are of the traditional type with areas of between 0.2 and 10.7 ha. The ploughing and weeding constitute the most frequent mechanical maintenance. A total of 36 varieties of mango trees were noted (including 31
\end{abstract}


local varieties). The zones are dominated by varieties such as Keitt and Kent. To this end, the inventory of fruit flora has made it possible to identify 46 species distributed in 42 genera held by 29 families in all the orchards. The typology, however, made it possible to define four groups of orchards.

Keywords: Characterization, Orchard, Mangifera indica, Casamance

\section{Introduction}

En Afrique de l'Ouest, la filière des fruits et légumes constitue l'un des secteurs agricoles qui connait une croissance rapide (Vayssières et al., 2008). Par ailleurs, la filière horticole a montré un fort dynamisme dans ces dix dernières années, grâce à la consolidation de pôles productifs stratégiques, à la hausse de la demande des marchés urbains nationaux et des marchés européens, et aux politiques nationales de développement agricole (FAO, 2006). La production de mangues occupe une place importante au Sénégal aussi bien pour le marché local que pour l'exportation. Elle demeure un maillon important de l'horticulture (Dias et al., 2012). En effet, la production fruitière annuelle sénégalaise était estimée en 2006 à 150.000 tonnes dont la mangue et les agrumes représentent respectivement $60 \%$ et $24 \%$ (FAO, 2006). En 2015, Cette production fruitière était évaluée à 246.500 tonnes dont plus de $50 \%$ (soit 20 milliards FCFA ) représentent la part de la mangue (RCSA, 2015) avec une augmentation de 96.500 tonnes en neufs ans. En effet, la production de la mangue constitue le moteur des cultures fruitières, suivie des agrumes $20,28 \%$, et de la banane $14,9 \%$.

Cependant, la production de mangues est menacée par les mouches des fruits. Ces insectes particulièrement Bactrocera dorsalis (Hendel), occasionnent d'importantes pertes de production de mangues. Suivant la zone et la période de production de mangues. Ces pertes peuvent varier de 30 à $50 \%$ dans la zone des Niayes voire 60\% en Casamance (Ternoy et al., 2006). En effet, la présence de ces insectes de quarantaine dans le territoire sénégalais serait à l'origine de 20 interceptions de mangues sur un total de 17168 tonnes exportées en 2017. Ces dernières se voient réduites à 10 en 2018 pour une quantité de 20000 tonnes exportées. Des études menées dans les Niayes et en Casamance ont fait état des relations existantes entre les caractéristiques des systèmes de production et la dynamique des mouches des fruits (Ndiaye et al., 2012 ; 2015 ; Diamé et al., 2015).

Afin de limiter les interceptions de mangues et les pertes de production causées par les mouches des fruits, une caractérisation des vergers à bases de manguiers a été initiée dans la zone des Niayes. Ensuite, dans le cadre du projet sous régional (PLMF/CEDEAO), cette caractérisation est élargie en Casamance qui est une importante zone de production de mangues malgré sa faible part sur les exportations. Ainsi, avec les faibles quantités de mangues 
exportées par rapport à sa production globale, la recherche des facteurs de vulnérabilité des vergers de la Casamance face à la mouche des fruits pourrait donner une nouvelle orientation pour améliorer la qualité de production.

\section{Matériel et Méthodes}

\subsection{Situation géographique de la zone d'étude}

Cette étude a été réalisée en Basse Casamance dans les Arrondissements de Kataba 1, Tendouck et Tenghory du Département de Bignona qui est situé entre $12^{\circ} 45^{\prime} 0^{\prime \prime}$ Nord et $16^{\circ} 30^{\prime} 0^{\prime \prime}$ Ouest. La zone est caractérisée par un climat de type soudanien côtier Sud (Sagna, 2005); et, une température et une pluviométrie moyennes annuelles respectivement évaluées, entre 1984 et 2015, à $27,10^{\circ} \mathrm{C}$ et 1322,66 mm (Dasylva et al., 2017).

La zone est limitée au nord par la Gambie, à l'ouest par l'Océan Atlantique, à l'Est par la région de Sédhiou et au Sud par le fleuve Casamance.

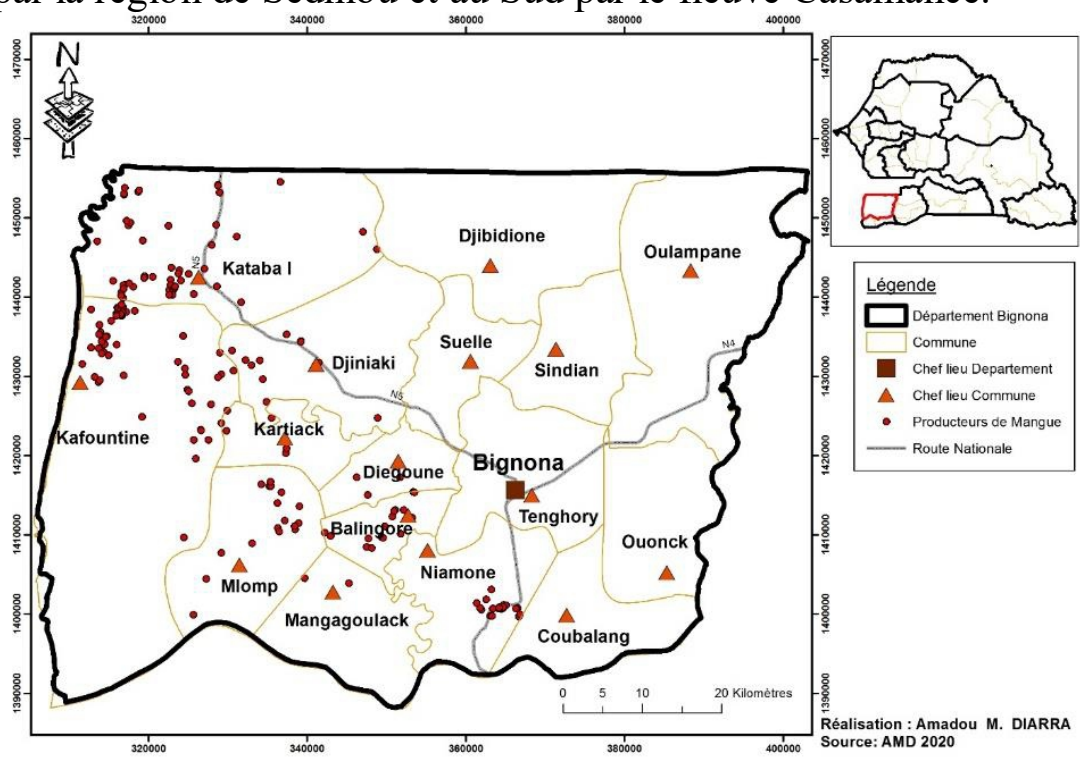

\subsection{Echantillonnage}

Sur la base d'une liste de 490 producteurs obtenue suite à la visite de prospection effectuée dans ces trois Arrondissements, 217 vergers (soit $44,28 \%$ ) ont été aléatoirement choisis pour un inventaire intégral des manguiers de par verger. Un échantillonnage systématique a été aussi appliqué pour un inventaire floristique des autres espèces fruitières associées. A la demande du projet sous régional de lutte contre la mouche des fruits (PLMF/CEDEAO), les investigations devraient porter sur des vergers potentiels à l'export avec au moins 80 vergers à Kataba 1 et à Tendouck concentrant l'essentiel de la production de mangues. Le choix de ces producteurs s'est fait sur la base d'un tirage au hasard sans remise des codes 
attribués à chaque verger. Le tableau 1 indique le nombre total de producteurs et de vergers échantillonnés par arrondissement.

Tableau 1. Nombre de producteurs et de vergers échantillonnés par Arrondissement

\begin{tabular}{|c|c|c|c|}
\hline Arrondissements & Vergers recensés & Vergers inventoriés & Pourcentage (\%) \\
\hline Kataba 1 & 211 & 90 & 42,65 \\
\hline Tendouck & 241 & 89 & 36,9 \\
\hline Tenghory & 38 & 38 & 100 \\
\hline Total & $\mathbf{4 9 0}$ & $\mathbf{2 1 7}$ & $\mathbf{4 4 , 2 8}$ \\
\hline
\end{tabular}

\subsection{Mesures de Paramètres dendrométriques}

La mesure des paramètres dendrométriques a été faite dans les plantations de manguiers grâce à un inventaire intégral des pieds de manguiers. Le diamètre des arbres a été mesuré à hauteur de poitrine $(1,30 \mathrm{~m}$ du sol) (DHP) à l'aide d'un compas forestier. Pour les arbres fourchus en dessous de la hauteur de poitrine, la formule suivante proposée par Thiombiano et al. (2015) a été appliquée pour obtenir le diamètre équivalent $D_{\text {é }}=\left(\sum d_{i}{ }^{2}\right)^{\left(\frac{1}{2}\right)}$ ( où $d_{i}$ est le diamètre à 1,30 mètre de la branche $i$, exprimé en centimètre. La hauteur de l'arbre a été estimée en mètre à l'aide d'un clisimètre suunto. Pour le diamètre du houppier deux mesures croisées (NordSud et Est-Ouest) ont été prises au niveau de chaque arbre à l'aide d'un ruban gradué de $30 \mathrm{~m}$ pour en dégager une moyenne. L'état du feuillage (note comprise entre les échelles 1 et $5 ; 1=$ feuillage peu dense $; 2=$ feuillage assez dense $; 3=$ feuillage moyennement dense $; 4=$ feuillage dense et $5=$ feuillage très dense) et la quantité de litière sous l'arbre (note comprise entre les échelles 1 et $3 ; 1=$ litière peu abondante ; $2=$ Litière moyennement abondante et $3=$ litière abondante) ont été appréciés.

Un échantillonnage systémique a été réalisé dans les vergers pour les espèces fruitières autres que le manguier cultivées ou spontanées comme Icacina senegelensis Juss, Anonna senegalensis Pers, Uvaria chamea P.Beauv, Saba senegalensis (A.DC.) etc. sur des placettes circulaires de $10 \mathrm{~m}$ de rayon soit $314 \mathrm{~m}^{2}$. Dans chaque verger, ces placettes ont été installées sur la plus importante toposéquence pour embrasser les différents faciès de végétation avec des distances de $20 \mathrm{~m}$ entre 2 centres de placettes successives. Ainsi l'inventaire floristique des fruitiers associés a été fait sur 868 placettes installées dans l'ensemble des vergers inventoriés. La moyenne du nombre d'espèces recensées dans les placettes est rapportée à la superficie du verger. Selon (Tandjiekpon, 2005), la hauteur de l'arbre, le diamètre du tronc et le diamètre du houppier sont des paramètres qui permettent de mieux cerner l'espace occupé réellement par les arbres pour une meilleure gestion à long terme des plantations.

Pour la détermination de la superficie, un tour complet du verger a été effectué avec le GPS. 


\subsection{Relevés des paramètres de la composante technique}

Pour compléter le travail de caractérisation des vergers, un questionnaire a été administré aux propriétaires de vergers ainsi que des focusgroupes auprès des dirigeants de l'Association des Producteurs Autonomes de Diouloulou et de ceux de la société «Saveurs du Sud» à l'aide d'un guide d'entretien. Des interviews semi- structurées ont été réalisées avec les GIE et les GPF rencontrés dans la zone d'étude.

\subsection{Traitement et analyses des données}

Une codification des questions a été d'abord réalisée avec le logiciel Sphinx plus 2000 avant d'effectuer les analyses grâce au même logiciel. Les données quantitatives et qualitatives ont été saisies sur le tableur Ms Excel. Ce tableur est utilisé pour les calculs et l'élaboration de tableaux et graphiques. Les tableaux croisés dynamiques ont été utilisés pour synthétiser les données. Des analyses multi variées (ACP) et des régressions simples ont été réalisées dans le cadre de la typologie des plantations et de la variation des paramètres à l'aide du logiciel R.3.5.2.

La diversité spécifique des plantations fruitières est analysée à l'aide des indices couramment utilisés, à savoir la richesse spécifique (nombre d'espèces), l'indice de diversité de Shannon et l'indice d'équitabilité de Pielou. Les deux derniers indices sont basés sur la notion de la régularité.

L'indice de diversité de Shannon (Shannon-Weaver): $\mathbf{H}=-\sum_{i}^{n} \boldsymbol{p}_{\boldsymbol{i}} \boldsymbol{l o g}_{2} \boldsymbol{p}_{\boldsymbol{i}}$ où: $\mathrm{H}=$ indice de diversité de Shannon

$\mathrm{Pi}=$ proportion des individus dans l'échantillon total qui appartiennent à l'espèce $i$.

Cet indice est basé sur la théorie de l'information. Il exprime l'importance relative du nombre d'espèces abondantes dans un milieu donné. L'indice est minimum quand tous les individus appartiennent à la même espèce. Il est maximal quand chaque individu représente une espèce distincte (Legendre \& Legendre, 1984). Exprimées en bit, les valeurs extrêmes sont comprises entre zéro (0) (diversité très faible) et 4,5 bits environ, ou exceptionnellement plus dans le cas des échantillons de grande taille dans les communautés complexes. La valeur de l'indice varie en fonction du nombre d'espèces présentes et de leurs proportions relatives. Ce qui rend plus exhaustif son usage pour la comparaison de deux milieux à richesse spécifique très différente. Ainsi, l'indice de régularité ou d'équitabilité (E) apparait plus intéressant pour comparer différents milieux (Devineau et al., 1984). L'indice d'équitabilité de Pielou (E) : $\mathbf{E}=\frac{H}{\log _{2} S}$ où :

$\mathrm{S}=$ nombre total des espèces dans le parc ;

$\mathrm{H}=$ indice de diversité de Shannon. 
La régularité correspond au rapport entre la diversité obtenue et la diversité maximale possible $\left(\log _{2} S\right)$ du nombre d'espèces $\mathrm{S}$. Elle varie entre zéro $(0)$ et un (1). L'équitabilité de Pielou (E) tend vers zéro lorsqu'il y a un phénomène de dominance. Elle tend vers un (1) lorsque la répartition des individus entre les espèces est régulière (Ramade, 1969).

Pour caractériser la distribution des superficies la formule de Freedman et Diaconis (1981) selon laquelle $\mathrm{h}=2(\mathrm{IQ}) \mathrm{n}^{-1 / 3}$ a été utilisée pour déterminer le nombre de classes.

$\mathrm{H}=$ nombre de classe ; IQ = distance inter quantile $; \mathrm{n}=$ nombre total d'individus.

La typologie des plantations est décrite sur la base des différents paramètres mesurés.

\section{Resultats Et Discussion}

\subsection{Resultats}

\subsubsection{Caractéristiques Des Vergers}

\subsubsection{Age Des Plantations}

La répartition des vergers par classe d'âges est présentée dans la Figure 1. Il ressort de cette répartition que $43,8 \%$ des vergers ont un âge compris entre 20 et 30 ans et $33,2 \%$ ont un âge compris entre 30 et 40 ans. Les vergers dont l'âge est inférieur ou égal à 20 ans représentent 13,9\%. L'âge moyen d'un verger de la zone est de 27 ans.

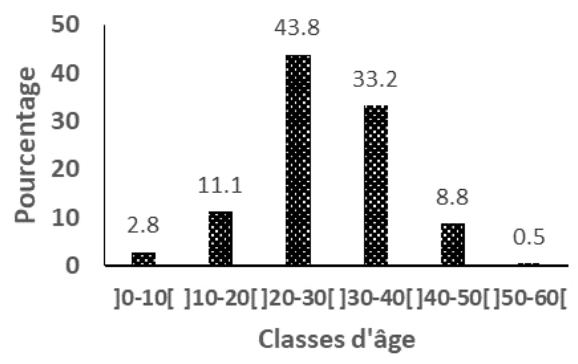

Figure 1. Répartition des vergers par classe d'âges.

\subsubsection{Superficies des plantations de manguiers}

Un total de 754,83 ha a été obtenu pour l'ensemble des vergers recensés. Les superficies occupées par un verger de manguiers varient entre 0,2 et 10,7 ha et la superficie moyenne exploitée par un planteur est de 1,72 ha. La Figure 2 représentant la répartition des plantations par classes de superficies indique que $46,29 \%$ des vergers ont une superficie comprise entre 0,9 et 1,81 ha et $21,75 \%$ ont une superficie comprise entre 0 et 0,9 ha. Par ailleurs, seulement $0,46 \%$ des vergers ont une superficie comprise entre 7 et 10 ha. Plus de $75 \%$ des vergers ont une superficie inférieure à 2 ha. 


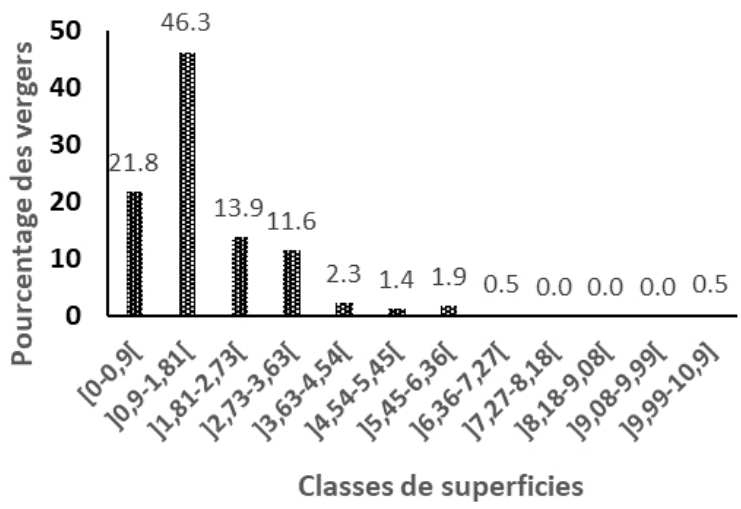

Figure 2. Répartition des plantations de manguiers en fonction des superficies

\subsubsection{Les Caractéristiques fonctionnelles des vergers}

Les caractéristiques fonctionnelles des vergers concernent les traitements phytosanitaires appliqués au sein des vergers, la fertilisation, l'irrigation, l'entretien des vergers, le dispositif de protection des vergers et les cultures annuelles associées aux plantations de manguiers. Le tableau 2 représente les pratiques qu'effectuent les producteurs au sein de leurs vergers.

Tableau 2. Proportion de producteurs par pratique de tenure de verger et par localité

\begin{tabular}{|l|r|r|r|r|r|r|}
\hline \multirow{2}{*}{ Pratiques effectuées } & \multicolumn{1}{|c|}{ Kataba 1 } & \multicolumn{1}{l|}{ Tendouck } & \multicolumn{2}{l|}{ Tenghory } \\
\cline { 2 - 7 } & Oui & Non & Oui & Non & \multicolumn{1}{l|}{ Oui } & \multicolumn{1}{l|}{ Non } \\
\hline Entretien & $93,25 \%$ & $6,75 \%$ & $94,38 \%$ & $5,62 \%$ & $97,36 \%$ & $2,64 \%$ \\
\hline Clôture & $31,46 \%$ & $68,54 \%$ & $43,89 \%$ & $56,11 \%$ & $71,05 \%$ & $29,95 \%$ \\
\hline Traitement phytosanitaire & $35,95 \%$ & $64,05 \%$ & $25,84 \%$ & $74,16 \%$ & $73,68 \%$ & $26,32 \%$ \\
\hline Irrigation & $5,61 \%$ & $94,39 \%$ & $0 \%$ & $100 \%$ & $0 \%$ & $100 \%$ \\
\hline Maraîchage & $14,6 \%$ & $85,4 \%$ & $8,98 \%$ & $91,02 \%$ & $26,31 \%$ & $73,69 \%$ \\
\hline Fertilisation & $53,93 \%$ & $46,07 \%$ & $61,79 \%$ & $38,21 \%$ & $2,61 \%$ & $97,37 \%$ \\
\hline
\end{tabular}

Les techniques de tenure pratiquées par les producteurs de la zone sont de types manuels. Le désherbage et le labour constituent les deux pratiques d'entretient mécaniques réalisées par les producteurs. De ce fait, ces entretiens sont plus récurrents dans l'Arrondissement de Tenghory (97,36\%) suivi de Tendouck (Blouf). De même, la protection des vergers $(71,05 \%)$ contre la divagation des animaux ainsi que l'utilisation des produits phytosanitaires $(73,68 \%)$ pour lutter contre la mouche des fruits sont plus fréquentes dans ce même Arrondissement (Tenghory). Le dispositif de protection le plus utilisé dans la zone couverte par l'étude est la haie morte constituée de piquets de bois mort tandis que le malatrap et le succès appât constituent les deux produits utilisés contre les mouches des fruits. Par conséquent, dans les Arrondissements de Tendouck et Tenghory, aucun verger n'est irrigué. C'est seulement dans l'Arrondissement de Kataba 1 où il a été noté 5,61\% de vergers irrigués. Ces derniers sont des plantations jeunes qui bénéficient de l'arrosage 
durant les premières années d'installation suite aux pratiques de maraîchage qui s'y effectuent. Par ailleurs l'association des cultures maraîchères aux plantations de manguiers $(26,31 \%)$ est pratiquée dans l'Arrondissement de Tenghory. La fertilisation quant à elle est effectuée dans $71,79 \%$ des vergers de l'Arrondissement de Tendouck et dans 53,93\% des plantations de l'Arrondissement de Kataba 1. En effet, l'apport de fertilisants aux manguiers se fait par technique de parcage des troupeaux de vaches dans les plantations après récolte.

\subsubsection{Composition floristique des vergers de manguiers}

\subsubsection{La diversité variétale}

Les vergers de manguiers sont caractérisés par la culture d'une diversité variétale au sein d'un même verger. Au total 36 variétés de mangues ont été répertoriées dans la zone dont cinq (5) améliorées et 31 variétés locales. En effet, toutes les variétés ne sont pas rencontrées dans toutes les zones. De ce fait, 24 variétés ont été recensées dans l'Arrondissement de Kataba 1, 14 à Tendouck et 19 à Tenghory (Tableau 3). Malgré cette diversité variétale élevée dans la zone, la moyenne variétale par verger est de 4.

Tableau 3. Répartition du nombre de variétés de manguiers par Arrondissement

\begin{tabular}{|c|c|}
\hline Arrondissements & Nombre de variétés \\
\hline Kataba 1 & 24 \\
\hline Tendouck & 14 \\
\hline Tenghory & 19 \\
\hline Total & $\mathbf{3 6}$ \\
\hline
\end{tabular}

Cependant, les tendances issues de la proportion des différentes variétés par localité, révèlent que la zone reste dominée par des variétés floridiennes telle que la Keitt et la Kent. La variété Keitt reste la plus représentée dans tous les trois Arrondissements (41,04\% à Kataba 1;58\% à Tendouck et $48 \%$ à Tenghory) (Figure 3). Du fait des reconversions variétales qui sont en train d'être effectuées, le pourcentage le plus élevé de la Kent (29,29\%) est répertorié dans l'Arrondissement de Kataba 1. Une forte conservation des variétés locales $(14,58 \%)$ autres que la Diourou a été constatée à Tenghory. Il est à signaler que toutes les variétés ne sont pas présentes dans toutes les localités. Ainsi, la variété Eldon est limitée à l'Arrondissement de Tendouck. Par ailleurs, les variétés Hadden, Tommy et Eldon restent faiblement représentées. 


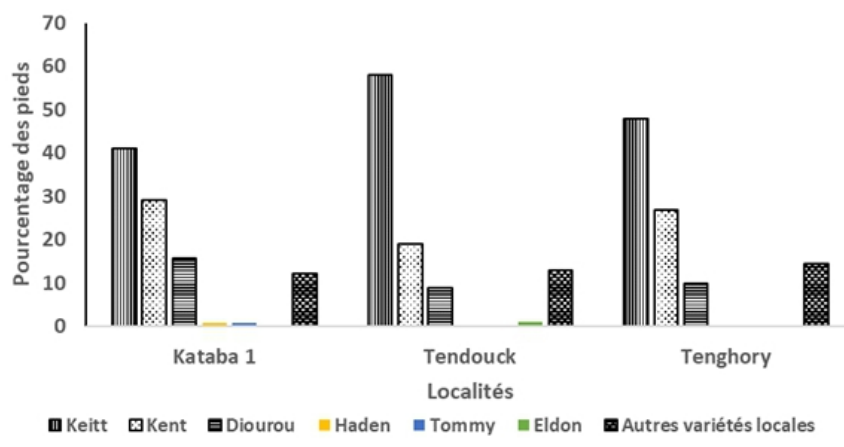

Figure 3. Proportion des différentes variétés de manguiers en fonction de la localité

\subsubsection{Les cultures annuelles associées aux plantations de manguiers}

Des cultures annuelles sont installées dans $71 \%$ des vergers de manguiers et seulement $29 \%$ des plantations sont en culture pure. La Figure 4 présente les proportions des vergers de manguiers où des cultures annuelles sont associées aux plantations. Dans les $71 \%$ des vergers associés à des cultures annuelles, des spéculations hivernales comme l'oseille (10\%), le niébé $(8 \%)$, l'arachide $(7 \%)$, le gombo $(7 \%)$, le manioc $(7 \%)$ et les pépinières de riz $(7 \%)$ sont les plus cultivées en association avec les manguiers.

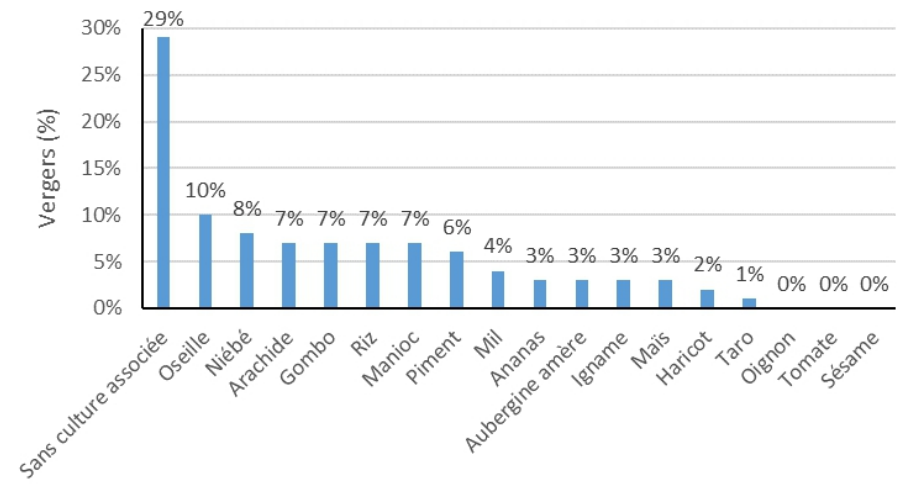

Cultures associées aux plantations

Figure 4. Proportion des vergers en fonction des cultures annuelles associées aux plantations

\subsubsection{Importance des espèces fruitières dans les vergers de manguiers}

$\mathrm{Au}$ total, 46 espèces fruitières réparties dans 42 genres appartenant à 29 familles ont été répertoriées. La famille des Anacardiaceae est la plus représentée $(48,28 \%$ ) avec des espèces de la famille comme Mangifera indica L., Anacardium occidentale L. et Spondias mombin L. Il s'ensuit les familles: des Rutaceae $(18,12 \%)$ représentée par Citrus limon (L.) Burm, Citrus sinensis (L.) Osbeck, Citrus grandis (L.) OSBECK, Citrus reticulata Blanco., des Icacinaceae (17,48\%) dont l'espèce Icacina senegalensis Juss., des Arecaceae (4,20\%) dont Borassus akeassii Bayton, Cocos nucifera L. et 
Elaeis guineensis Jacq., des Annonaceae (2,17\%) dont Annona muricata L., Annona senegalensis Pers. et Uvaria chamea P.Beauv, des Apocynaceae (1,54\%) dont Landolphia heudolotii A.DC., Landolphia dulcis Sabine et Saba senegalensis (A.DC.) Pichon.

Les paramètres de diversité des vergers sont indiqués dans le tableau 4 cidessous.

Tableau 4. Paramètres de diversité des fruitiers cohabitant dans les plantations des différents Arrondissements

\begin{tabular}{|c|c|c|c|}
\hline Variables & Kataba 1 & Tendouck & Tenghory \\
\hline Richesse spécifique & 46 & 22 & 28 \\
\hline Diversité de Shannon H' (bit) & 2,16 & 1,85 & 2,17 \\
\hline Equitabilité de Pielou E & 0,39 & 0,41 & 0,45 \\
\hline
\end{tabular}

Le nombre d'espèces est plus important dans l'Arrondissement de Kataba 1 (46 espèces) que dans les deux autres. De même, ce dernier renferme des vergers plus diversifiés en espèces fruitières $\left(H^{\prime}=2,16\right)$ avec celui de Tenghory $\left(H^{\prime}=2,17\right)$ que l'Arrondissement de Tendouck. En revanche, cette diversité révèle un phénomène de dominance des individus d'une espèce par rapport aux autres dans tous les arrondissements $(E=0,39$ et 0,45$)$. Pour un nombre d'espèces plus important dans l'Arrondissement de Kataba1, la répartition des individus est sensiblement la même que celui Tendouck.

\subsubsection{Structuration et typologie des vergers de manguiers}

\subsubsection{La densité des plantations de manguiers}

Les différents vergers ont des densités très variables allant de $3 \mathrm{ind} / \mathrm{ha}$ (Kataba 1) à $252 \mathrm{ind} / \mathrm{ha}$ (Tendouck). La densité moyenne des plantations de la zone est de $49 \mathrm{ind} / \mathrm{ha}$. Cette densité est plus importante dans les Arrondissements de Tendouck et Tenghory avec respectivement 65 et 48 ind /ha (Figure 5). L'Arrondissement de Kataba 1 présente les plus petites densités avec une moyenne de 32 ind /ha.

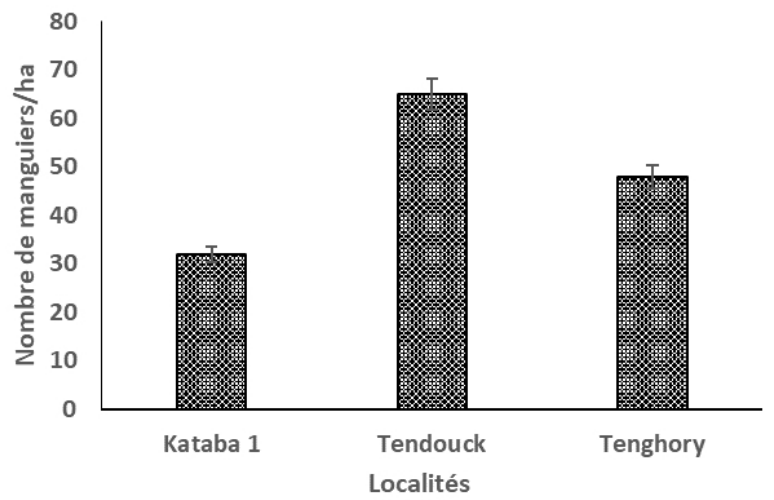

Figure 5. Répartition des densités moyennes des manguiers par Arrondissement 


\subsubsection{Variation des paramètres dendrométriques}

La variation du diamètre à hauteur de poitrine (DHP en $\mathrm{cm}$ ), de la hauteur (en $\mathrm{m}$ ) ainsi que du diamètre du houppier (DH en $\mathrm{m}$ ) en fonction des localités est illustrée par la Figure 6. Il ressort de l'analyse des boxplots issus de la régression de «Poisson » que la corrélation entre les paramètres mesurés et les localités est significative ( $\mathrm{p}$-value $=0,002<$ alpha $=0,05$ ). A cet effet, les paramètres mesurés varient d'un Arrondissement à un autre, donc les plantations sont hétérogènes. Ainsi, le diamètre à hauteur de poitrine diminue de $-0,09 \mathrm{~cm}$, la hauteur de $-1,23 \mathrm{~m}$ et le diamètre du houppier de $-1,23 \mathrm{~m}$. En revanche, les boxplots indiquent aussi que l'Arrondissement de Tenghory renferme les manguiers les plus gros diamètres de troncs, de hauteurs plus importantes et des houppiers plus développés comparés aux autres arrondissements.
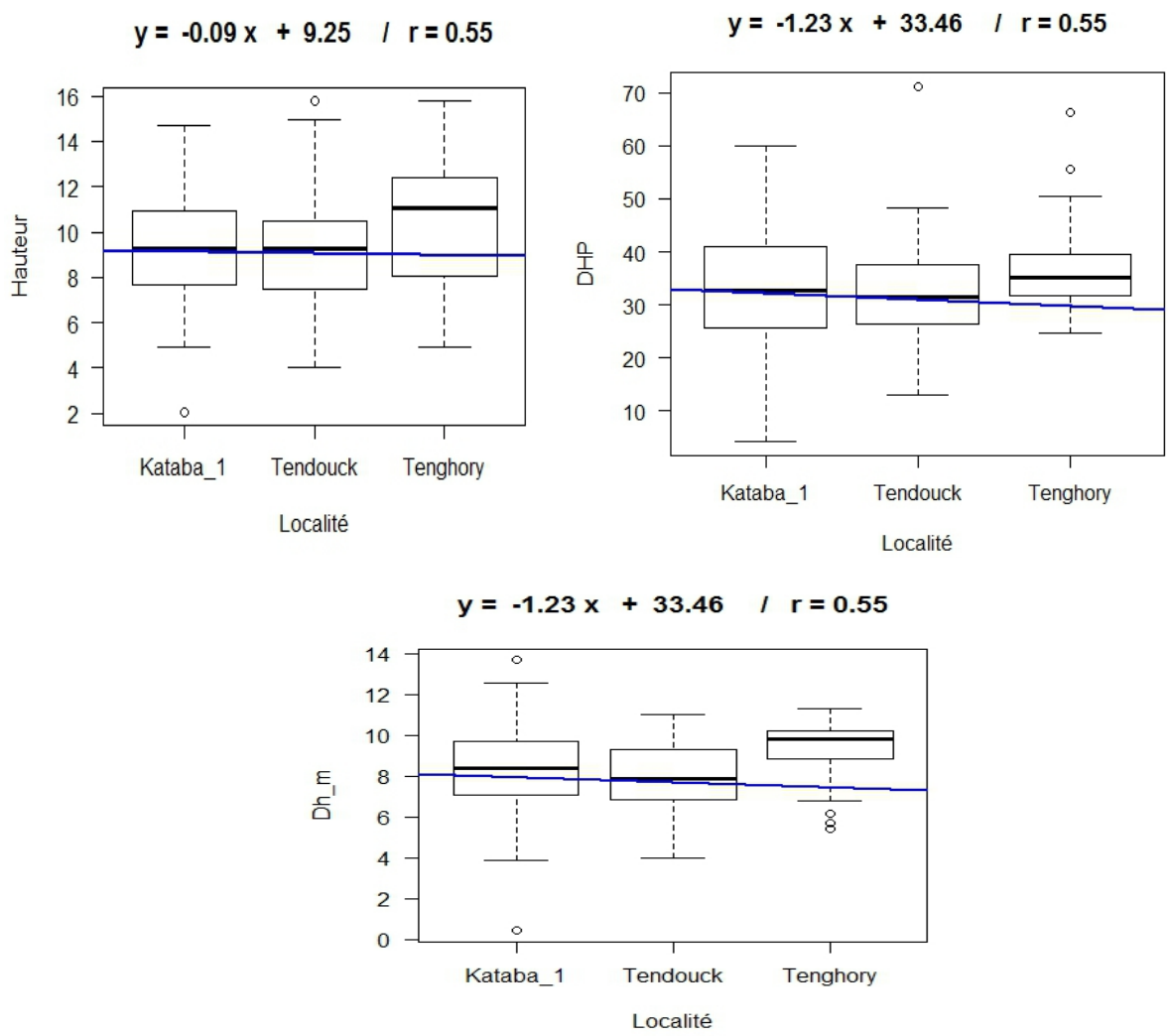

Figure 6. Variation des paramètres dendrométriques mesurés selon l'Arrondissement

Par conséquent, ces paramètres ne révèlent pas une même tendance selon leur variation en fonction de l'âge. La Figure 7 montre le comportement des différentes variables dendrométriques mesurées en fonction de l'âge des plantations. A l'issue de l'analyse des graphiques de la régression linéaire, il 
ressort que la corrélation entre les variables mesurées et l'âge des plantations est significative ( $\mathrm{p}$-value $=0,002<$ alpha $=0,05)$. A chaque fois que l'âge varie d'une unité, le diamètre des manguiers croît de $0,12 \mathrm{~cm}$, la hauteur de $0,01 \mathrm{~m}$ et le diamètre du houppier de $0,02 \mathrm{~m}$.
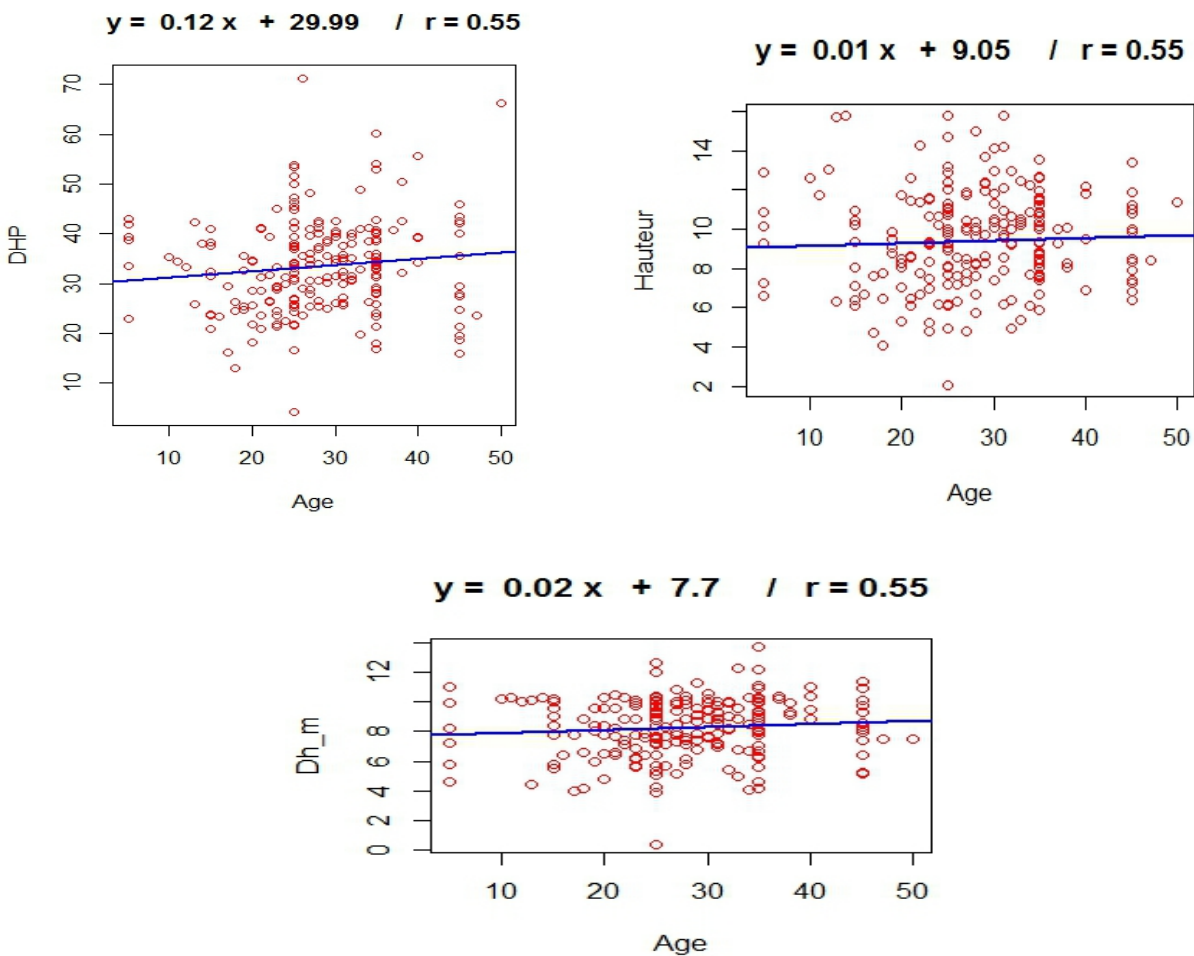

Figure 7. Variation du houppier, du diamètre à hauteur de poitrine et de la hauteur des manguiers en fonction de l'âge des plantations

\subsubsection{Typologie des plantations de manguiers}

La typologie de la plantation est faite sur la base des différentes variables quantitatives caractéristiques des vergers de la zone. Au seuil de signification Alpha $=0,050$, il existe des corrélations significatives ( $\mathrm{p}$-value < $0,0001)$ entre les variables telles la densité du feuillage, le diamètre du tronc, le diamètre du houppier, le nombre de variétés dans le verger, la superficie, la densité, la hauteur de l'arbre.

La matrice de corrélation a permis de définir les plantations qui ont sensiblement les mêmes caractéristiques. Ainsi, l'analyse en composantes principales (ACP) a permis de regrouper les vergers en quatre groupes en fonction de leurs similarités caractéristiques (Figure 8). Elle informe que les paramètres comme 'Feuillage', 'DHP', 'Dh_m' et la 'Hauteur' sont portés par l'axe F1. Les autres paramètres sont portés par F2 avec une corrélation 
négative entre la 'Densité' d'une part et la 'Superficie', l' 'Age', le 'Nombre de variétés' et la litière constituée par les 'Feuilles_au_sol' d'autre part.

Le groupe A est caractérisé par des vergers d'âge avancé s'étendant sur de grandes superficies avec une diversité variétale importe et une litière abondante. Quant au groupe B, il est caractérisé par des manguiers à gros diamètre de tronc, un feuillage dense, un diamètre moyen du houppier important et une hauteur moyenne des arbres élevée. Les vergers englobant les plantations à forte densité sont rassemblés au niveau du groupe C. Par ailleurs, les vergers renfermant des manguiers à faible diamètre de tronc, à faible diamètre de houppier, à hauteur faible et à faible densité de feuillage sont ceux du groupe D.

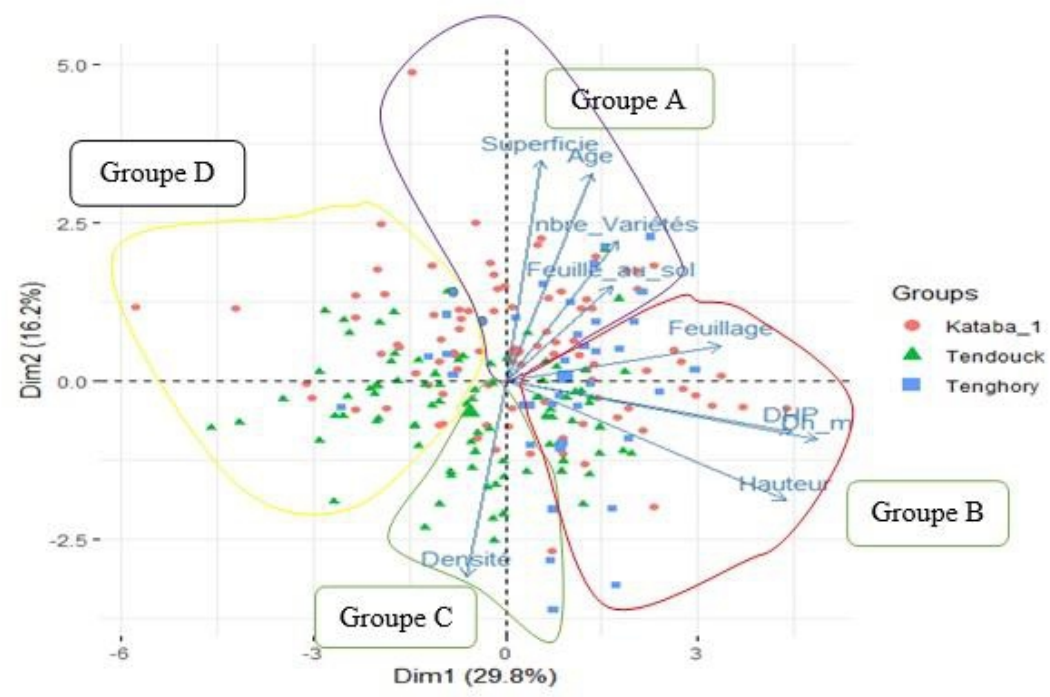

Figure 8. Les différents groupes de vergers issus de la typologie des plantations.

\subsection{Discussion}

Les plantations de la zone (86,1\%) sont âgées de plus de 20 ans. L'âge moyen d'un verger de la zone est de 27 ans. En effet, pour les vergers âgés, beaucoup de producteurs ne connaissent pas la date exacte de plantation. Un producteur a affirmé que son verger est âgé de plus de 100 ans. Ces plantations ne massifient pas la production de mangues car plus le manguier est vieux, moins il produit (Davenport, 2009). De ce fait, un manguier peut vivre plusieurs centaines d'années et avec une production faible de taille arrondie des mangues (Mukherjee \& Litz, 2009).

La superficie moyenne emblavée pour un verger de manguiers est pratiquement petite $(1,72 \mathrm{ha})$. En effet, les superficies des plantations de la zone varient entre 0,2 ha et 10,7 ha. Cette fourchette correspond à la tendance générale au niveau du pays et en Afrique de l'Ouest. À cet égard, Vannière et 
al. (2004a) stipulent qu'à part certains manguiers trouvés isolés ou mélangés à d'autres espèces fruitières, l'essentiel des vergers de manguiers exploités en Afrique de l'Ouest a moins de 10 ha. Blondeau (2007) a montré que les producteurs sénégalais disposent de petites et moyennes exploitations de superficie variant pour la majorité entre 1 et 10ha. Ces petites superficies révéleraient la nature des plantations qui sont constituées de vergers de case, de type traditionnel, non préparés pour l'exportation de la mangue. Abordant dans le même sens ASEPEX (2011) affirme que globalement, les plantations villageoises occupent la superficie la plus importante, leurs rendements sont faibles et les superficies individuelles sont modestes.

L'entretien le plus couramment réalisé est de type mécanique. L'entretien sanitaire pour les manguiers est quasi absent dans la zone ; ce qui pourrait favoriser les attaques des ennemis des fruits. Les manguiers malades sont laissés à leur sort. Le désherbage et le labour constituent les pratiques d'entretien les plus réalisées. Le désherbage est le plus souvent réalisé en période de premiers fruits pour faciliter les récoltes. Par ailleurs, Ouédraogo (2007) a montré que le manque d'entretien des parcelles dans les vergers de manguiers du Kénédougou favorise les attaques des ennemis des fruits. La taille d'entretien est pratiquée dans les vergers pour limiter la croissance en hauteur des arbres. Elle permet aux fruits colorées de mieux manifester leur coloration et la taille de régénération est le plus souvent suivie d'une reconversion variétale dite surgreffage (Guira, 2002). Les clôtures effectuées dans les vergers assureraient leur protection contre la divagation des animaux. Les haies mortes (en piquets de bois) et les dispositifs en piquets de bois + Euphorbia lathyris L. sont les plus utilisées. En effet, les vergers de la région de Korhogo (Côte d'Ivoire) sont clos avec des haies mortes ou vivantes, reliées par des fils de fer barbelés (Vannière et al., 2004b). Ce choix serait dû au bois qui constitue la ressource la plus accessible pour les populations mais aussi permettrait la pénétration du vent dans les plantations. Les haies vives clôturant complètement les plantations sont rares alors que dans les Niayes, les vergers sont principalement associés à des haies avec des espèces de bornage et des espèces défensives (Grechi et al., 2013). La quasi-totalité des vergers de la zone ne sont pas irrigués. Les manguiers jeunes bénéficient donc de l'eau d'arrosage des cultures maraichères associées aux plantations des jeunes vergers. Cette situation dénote du caractère traditionnel du système de production tandis que la production sénégalaise est composée de vergers de type industriels, de vergers maîtrisés et de plantations villageoises (ASEPEX, 2011). Abordant dans le même sens, Vannière et al. (2013) indiquent que le puissant système racinaire du manguier lui permet de s'alimenter directement dans les nappes peu profondes. Dans ce cas, les vergers ne sont pas irrigués. En plus, les manguiers ne seraient arrosés que par les eaux de pluie depuis leur implantation au mois de juin. Et jusqu' au $9^{\circ}$ ou $10^{\circ}$ de latitude Nord, les arbres 
plantés en mai ou juin parviennent à traverser la saison sèche suivante sans trop souffrir (Vannière et $a l ., 2004 b$ ). Le fertilisant le plus utilisé dans la zone est le fumier provenant du parcage des troupeaux de bovins au sein des plantations de manguiers. Les plantations bénéficient donc du fumier des bovins, attachés dans les vergers au niveau des pieds de manguiers durant presque toute l'année. En plus, le dépôt des résidus de récolte (fanes d'arachide), ou des ordures ménagères aux pieds des arbres fruitiers (manguiers et agrumes), constitue pour l'essentiel l'amendement organique.

Ces plantations sont dominées par Mangifera indica dans les deux Arrondissements (Tenghory et Tendouk) et par Citrus sinensis dans l'Arrondissement de Kataba 1. Cela s'explique par le fait que seules les espèces utiles sont épargnées lors des défrichements et entretenues dans les plantations, ce qui réduit la diversité naturelle (Ndiaye et al., 2017). La conservation de ces espèces fruitières dans les plantations de manguiers favoriserait l'implantation des mouches (Ndiaye, 2014). Des études réalisées au Bénin ont montré que les deux taux d'infestation les plus importants concernent la prune africaine (S. birrea) et l'annone sauvage (A. senegalensis) qui diffèrent significativement des taux d'infestation enregistrés pour les fruitiers cultivés (manguier et goyavier) qui ont des taux déjà importants (Vayssières et al., 2010). Aussi, sur Soixante-trois espèces de plantes de trente-neuf familles échantillonnées à l'Ouest du Burkina Faso, huit espèces (Annona senegalensis Pers, Ficus ingens (Miq.) Miq., Landolphia heudoletii A. DC., Sarcocephalus latifolius (Smith) Bruce, Saba senegalensis (A. DC.) Pichon, Sclerocarya birrea (A. Rich) Hochst, Strychnos innocua Del. et Vitellaria paradoxa Gaertn. F. appartenant à huit familles différentes ont été identifiées comme hôtes des mouches des fruits (Ouedraogo et al., 2008).

On mesure ainsi toute l'importance des fruits «sauvages» dans le développement des populations de mouches des fruits à côté des vergers de fruitiers cultivés. Les fruits n'arrivant pas à maturité en même temps, ces dernières trouveraient un lieu de refuge lors de la période défavorable (fin production de mangues). En considérant l'étalement des périodes de fructification de ces fruitiers locaux (hôtes) et le régime polyphage de ces ravageurs la protection phytosanitaire est à planifier à l'échelle des bassins de production regroupant à la fois les fruitiers spontanés et les fruitiers cultivés (Vayssières et $a l ., 2010$ ). La valeur des différents indices de diversité varie d'une commune à une autre. L'indice de Shannon permet la comparaison de deux milieux en terme de diversité floristique (Sonké, 1998). Ses valeurs importantes dans les localités (Arrondissement de Kataba 1) témoignent de la conversion de certains massifs forestiers en plantations de manguiers plus agrumes pures. Aujourd'hui, la culture des manguiers et des agrumes est responsable de la disparition de beaucoup d'espèces végétales dans cette zone. Ce qui se traduit par une faible présence d'espèces fruitières sauvages dans les 
plantations. Donc, les manguiers et les agrumes occupent la quasi-totalité des plantations de la zone souvent en «plantations mixtes ». En effet, les vergers mixtes (dominante manguiers mais avec des agrumes) ont généralement des populations de Bactrocera dorsalis plus importantes que les vergers homogènes (Vayssières et al., 2009). La zone reste dominée par des variétés floridiennes comme la Keitt et la Kent. Si dans les années 80, les «mangots » et la variété Amélie composaient $90 \%$ des vergers de manguiers en Afrique de l'Ouest, on observe aujourd'hui une domination des variétés floridiennes telles que Kent et Keitt, et à un degré moindre, Amélie (Saiah et al., 2012).

La pratique du surgreffage et la taille d'entretien influent beaucoup sur la variation de la hauteur dans la zone. En effet, le manguier le plus haut des sujets mesure $26 \mathrm{~m}$ tandis que cette espèce peut atteindre $30 \mathrm{~m}$ de hauteur en Côte d'Ivoire (Huguenin, 2010). La hauteur moyenne des manguiers dans cette zone est de $10,9 \mathrm{~m}$. Le diamètre du plus gros tronc de manguier mesure $105 \mathrm{~cm}$. Ce résultat corrobore celui de Daniel-yves (2002) qui disait que le fût du manguier est érigé et peut présenter un diamètre avoisinant le mètre. Le diamètre moyen des manguiers de la zone d'étude est de $36,3 \mathrm{~cm}$. La croissance latérale entraine une grande occupation du sol par le manguier au détriment des cultures. Dans la zone d'étude, les pratiques sylvicoles comme la taille d'entretien et le surgreffage font que le houppier des arbres ne peut être corrélé à l'âge de la plantation. Néanmoins, le diamètre moyen du houppier est de 9,24 m alors que selon De Laroussilhe (1980), il peut être de $10 \mathrm{~m}$ en moyenne.

\section{Conclusion}

La présente étude menée dans le département de Bignona a pour objectif de faire la caractérisation végétale et la typologie des vergers de manguiers. Cette étude a permis de connaitre les systèmes de production de mangues dans le terroir. Les résultats obtenus dans le cadre de la présente étude constituent une étape importante dans la connaissance des caractéristiques des vergers de la Casamance. Les plantations ont été regroupées dans quatre groupes compte tenu des différentes variables analysées. Les indices de diversité calculés sur la base des fruitiers varient d'un Arrondissement à un autre. Les vergers de la zone reflètent d'un manque d'entretien de la part des producteurs. En plus, les résultats de l'enquête ont montré que les plantations sont en majorité de vergers de type traditionnel. Cette étude a révélé aussi que la zone est dominée par des variétés floridiennes, propices à l'exportation. Les caractéristiques des vergers étudiées à travers les paramètres de végétation ont montré que la croissance de ces derniers n'est pas corrélée à l'âge des plantations.

Cependant, l'exportation et l'évacuation de la mangue restent la préoccupation majeure des producteurs. Les mouches des fruits constituent la 
principale entrave à l'exportation de la mangue malgré les efforts de lutte consentis par les producteurs. Les principales difficultés que rencontrent les producteurs de la zone, restent l'encadrement limité, le faible niveau d'organisation de la filière et la méconnaissance des méthodes de lutte phytosanitaire. En effet, des initiatives sont en train d'être menées par la sousrégion en général et le gouvernement sénégalais en particulier dans la lutte contre les mouches des fruits en passant par la modernisation des vergers.

En perspective, cette étude est une phase appropriée pour améliorer et diversifier la production de la mangue. Il serait donc intéressant d'élargir le champ de l'étude en Haute et Moyenne Casamance pour aboutir à une typologie des vergers à base de manguiers qui permettrait de capitaliser les différentes expériences et améliorer la filière mangue. Elle permettrait d'évaluer la rentabilité et la durabilité de cette filière en vue d'évaluer sa réelle contribution dans le développement économique et social de la Casamance en générale et des producteurs en particulier.

\section{References:}

1. ASEPEX (2011). Etude des marchés d'exportation de la mangue. Rapport Ministère du Commerce du Sénégal. 25p.

2. Blondeau, A. (2007). Etude d'impact des normes privées et réglementations européennes sur les filières horticoles sénégalaises: cas de la mangue et du haricot vert. Mémoire D'ingénieur Agronome , Sup Agro IRC Montpellier. 174p.

3. Daniel-yves, A. (2002). «Initiation à l'agroforesterie en zone sahélienne: Les arbres des champs du Plateau Central au Burkina Faso», Paris (FRA) ; Paris : IRD Editions ; Karthala, 220 p. ISBN 27099-1475-1.

4. Dasylva, M., Ndour, N., Ndiaye, O. \& Sambou, B. (2017). Analyse de la flore, de la végétation ligneuse et des fonctions des vallées en zone péri-urbaine post-conflit (Ziguinchor, Sénégal). Int. J. Biol. Chem. Sci. 11(1): 360-377,

5. Davenport, T.L. (2009). Reproductive physiology. UK CABI 97-169.

6. De Laroussilhe, F. (1980). Le Manguier: Techniques Agricoles et Productions Tropicales (Maisonneuve and Larose (Edition), Paris, France) ,312p.

7. Devineau, J.-L., Lecordier, C., \& Vuattoux, R. (1984). Evolution de la diversité spécifique du peuplement ligneux dans une succession préforestière de colonisation d'une savane protégée des feux (Lamto, Côte-d'Ivoire). Candollea 39, 103-134.

8. Diamé, L., Grechi, I., Rey, J.Y., Sané, C.A.B., Diatta, P., Vayssières, J.F., Yasmine, A., Bon, H.D., \& Diarra, K. (2015). Influence of Oecophylla longinoda Latreille, 1802 (Hymenoptera: Formicidae) on 
mango infestation by Bactrocera dorsalis (Hendel)(Diptera: Tephritidae) in relation to Senegalese orchard design and management practices. Afr. Entomol. 23, 294-305.

9. Dias, N.D.S., Zanetti, R., Santos, M.S., Gomes, M.F., Peñaflor, V., Broglio, S.M.F., \& Delabie, J.H.C. (2012). The impact of coffee and pasture agriculture on predatory and omnivorous leaf-litter ants, Journal of Insect Science 13, 1-11.

10. FAO (2006). FAO, 2006. World Base Ref. Soil Resour. Rep. World Soil Resour. FAO Rome Italy.

11. Freedman, D. \& Diaconis, P. (1981). On this histogram as a density estimator: L2 theory. Zeit Wahr Ver Geb 57, 453-476.

12. Grechi, I., Sane, C.A.B., Diame, L., De Bon, H., Benneveau, A., Michels, T., Huguenin, V., Malézieux, E., Diarra, K., \& Rey, J.Y. (2013). Mango-based orchards in Senegal: diversity of design and management patterns $=$ Vergers à base de manguiers au Sénégal: diversité des modèles de conception et de gestion Fruits 68, 447-466.

13. Guira, M. (2002). Rapport d'activités de recherche en arboriculture fruitière. (Burkina Faso: INERA 1), Document interne. 15p.

14. Huguenin, V. (2010). Influence des pratiques agricoles, de la structure du verger et de son environnement immédiat, sur la mouche du fruit, Mémoire de Master 2 «Gestion de la biodiversité et des Ecosystèmes Continentaux et Côtiers », Université de Lille 1. 66p.

15. Legendre, L. \& Legendre, P. (1984). Ecologie Numérique: 2. La structure des données écologiques (Masson,). 2icme Cd. Tome 2. Masson. Paris et les presses de l'Université du Québec, 260p.

16. Mukherjee, S.K. \& Litz, R.E. (2009). Introduction: Botany and importance. In: Litz RE, ed. The Mango: Botany, Production and Uses. 2nd Ed. 1-18.

17. Ndiaye, O. (2014). Interactions plantes hôtes, mouches des fruits et parasitoïdes dans les Niayes et la Basse Casamance. Thèse de Doctorat, Université Cheikh Anta Diop.120p.

18. Ndiaye, O., Ndiaye, S., Djiba, S., Ba, C.T., Vaughan, L., Rey, J-Y. \& Vayssières, J-F. (2015). Preliminary surveys after release of the fruit fly parositoid Fopius arisanus sonan (Hymenoptera Braconidae) in mango production systems in Casamance (Senegal). Fruits 70 (2): 9199.

19. Ndiaye, O., Vayssieres, J.-F., Rey, J.Y., Ndiaye, S., Diedhiou, P.M., Ba, C.T., \& Diatta, P. (2012). Seasonality and range of fruit fly (Diptera: Tephritidae) host plants in orchards in Niayes and the Thiès Plateau (Senegal). Fruits 67, 311-331..

20. Ndiaye, S., Charahabil, M.M., \& Diatta, M. (2017). Caractérisation des Plantations à base d'anacardier (Anacardium occidentale L.) dans le 
Balantacounda: cas des communes de Kaour, Goudomp et Djibanar (Casamance/Sénégal). Eur. Sci. J. ESJ 13.

21. Ouédraogo, S.N. (2007). Étude des attaques des mouches de fruits (Diptera tephritidae) sur la mangue dans la province du Kénédougou (Ouest du Burkina Faso). Mém. Diplôme D’étude Approfondie DEA En Gest. Intégrée Ressour. Nat. GIRN. Institut Développement Rural Université Polytechnique Bobo-Dioulasso. 57p.

22. Ouedraogo, S.N., Vayssieres, J.F., Rémy, A.D., \& Rouland-lefevre, C. (2008). Fruitiers locaux hôtes des mouches des fruits (diptera: tephritidae) de la mangue dans l'ouest du burkina faso: identification et taux d'infestation. In : Regional workshop on the "Potential role of local fruit tree and other food tree species for nutrition, poverty alleviation and biodiversity conservation in Sub-Saharan Africa: Factors to consider", 23-16 February 2009, Ouagadougou, Burkina Faso. s.l. : s.n., 23 p.

23. Ramade, F. (1969). Eléments d'écologie. Naturalist 83, 91-112.

24. RCSA (2015). Version finale | Direction de l'Analyse, de la Prévision et des Statistiques Agricoles - DAPSA, htt/www.dapsa.gouv.sn.le 04/11/2016.

25. Saiah, C., Parrot, L., Requier-Desjardins, D., Rey, J.-Y., Temple, L., Vayssières, J.-F., Vannière, H., Dia, B.K., \& Diarra, K. (2012) Quel impact des activités du CIRAD sur la filière mangue en Afrique de l'Ouest?, Rapport de stage au CIRAD du 1er Mars 2012 - 31 Août 2012. 192p.

26. Sagna, P. (2005). Dynamique du climat et son évolution récente dans la partie ouest de l'Afrique occidentale, Thèse de doctorat d'Etat ES Lettre, UCAD, Faculté des lettre et Sciences Humaine, Département de Géographie, 790p.

27. Sonké, B. (1998). «Etudes floristiques et structurales des forêts de la réserve de faune du Dja (Cameroun) ». Thèse de doctorat. Université Libre, 266p.

28. Tandjiekpon, A.M. (2005). Caractérisation du système agroforestier à base d'anacardier (Anacardium occidentale Linnaeus) en zone de savane au Bénin. Mémoire du diplôme d'Etudes Approfondies (DEA), FLASH. Université d'Abomey-Calavi, 122p.

29. Ternoy, J., Poublanc, C., Diop, M., \& Nugawela, P. (2006). La chaine de valeurs mangue au senegal:analyse et cadre strategique d'initiatives pour la croissance de la filière. Programme USAID / Croissance Economique, BDS Component Report, 271, 91p.

30. Thiombiano, A., Glele Kakaï, R., Bayen, P., Boussim, J. I. \& Mahamane, A. (2015). Méthodes et dispositifs d'inventaires forestiers en Afrique de l'ouest: état des lieux et propositions pour une 
harmonisation: état des lieux. In Annales des Sciences Agronomiques 19 - spécial Projet Undesert-UE : 15-31 ISSN 1659-5009

31. Vannière, H., Didier, C., Rey, J.-Y., Diallo, T.M., Kéita, S., \& Sangaré, M. (2004a). La mangue en Afrique de l'Ouest francophone: les systèmes de production et les itinéraires techniques. Fruits 59, 383398.

32. Vannière, H., Didier, C., Rey, J.Y., Diallo, T.M., Kéita, S., \& Sangaré, M. (2004b). La mangue en Afrique de l'Ouest francophone: les systèmes de production et les itinéraires techniques. Fruits 59, 383398.

33. Vannière, H., Rey, J.-Y., Vayssières, J.-F., \& Maraite, H. (2013). PIPItinéraire technique mangue (Mangifera indica) Bruxelles : COLEACP, $87 \mathrm{p}$.

34. Vayssières, J.F., Korie, S., Coulibaly, T., Temple, L., \& Boueyi, S. (2008). The mango tree in northern Benin (1): cultivar inventory, yield assessment, early infested stages of mangos and economic loss due to the fruit fly (Diptera Tephritidae). Fruits 63, 335-348.

35. Vayssières, J.-F., Rey, J.Y., Lyannaz, J.P., Sinzogan, A., Mana, P., Marone, D., Ndiaye, O., Ngamo, L., \& Ladang, D. (2009). Fluctuations spatiotemporelles des populations de Bactrocera invadens (Diptera, Tephritidae) au niveau des manguiers du Bénin, du Cameroun et du Sénégal, Coll. Prasac Ardesac Garoua Cameroun. 2p.

36. Vayssières, J.-F., Sinzogan, A., Adandonon, A., Ayegnon, D., Ouagoussounon, I., \& Modjibou, S. (2010). Principaux fruitiers locaux des zones guinéo-soudaniennes du Bénin: inventaire, période de production et dégâts dus aux mouches des fruits. Fruit Veg. Cereal Sci Biotechnol Glob. Sci Book Ltd Spec 42-46. 